\title{
The Promotion of the Efficiency of Organic Photovoltaic Devices by Addition of Anisotropic CdSe Nanocrystals
}

\author{
Shu-Ru Chung, ${ }^{1}$ Hong-Shuo Chen, ${ }^{2}$ Chen-Yu Chien, ${ }^{2}$ Meng-Yi Bai, ${ }^{3}$ and Kuan-Wen Wang ${ }^{2}$ \\ ${ }^{1}$ Department of Materials Science and Engineering, National Formosa University, Yunlin 63201, Taiwan \\ ${ }^{2}$ Institute of Materials Science and Engineering, National Central University, Taoyuan 32001, Taiwan \\ ${ }^{3}$ Graduate Institute of Biomedical Engineering, National Taiwan University of Science and Technology, Taipei 10607, Taiwan
}

Correspondence should be addressed to Shu-Ru Chung; srchung@nfu.edu.tw and Kuan-Wen Wang; kuanwen.wang@gmail.com

Received 13 March 2014; Revised 28 April 2014; Accepted 30 April 2014; Published 21 May 2014

Academic Editor: Aung Ko Ko Kyaw

Copyright (C) 2014 Shu-Ru Chung et al. This is an open access article distributed under the Creative Commons Attribution License, which permits unrestricted use, distribution, and reproduction in any medium, provided the original work is properly cited.

CdSe nanocrystals (NCs) with different morphologies have been synthesized and applied as the acceptor in the active layer of the organic photovoltaic (OPV) devices. CdSe tetrapod (TP)/nanorod (NR) with zinc-blended seeds and wurtzite arms is prepared by seed growth method and mixed with poly(3-hexylthiophene) (P3HT): [6,6]-phenyl-C61-butyric acid methyl ester (PCBM). When the concentrations of CdSe in P3HT: PCBM system are $50 \mathrm{wt} \%$ optimally, the efficiency can be promoted about $4.3 \%$, suggesting that an enhancement of $13.2 \%$ can be obtained and the addition of anisotropic CdSe NCs content in the active layer can be beneficial for the transport of electrons and light absorption in the OPV devices.

\section{Introduction}

Polymeric solar cells have attracted much attention during the last years due to their lower fabrication cost and possibility of using flexible substrates [1]. However, their efficiency is still about $9 \%$, because various factors, such as photo absorption window and coefficient, excitons separation and transportation, and excitons diffusion length have not been optimized fully [2-6]. Moreover, the active layer morphology related to blend preparation and annealing processes can also influence the efficiency significantly. Park et al. have reported that the power conversion efficiency increases from 0.5 to $2.6 \%$ for a single junction cell by carrying out thermal annealing of P3HT:PCBM cell due to the enhanced spectral absorption and decrease in charge carrier recombination [7]. Inorganic semiconductor nanocrystals (NCs), due to their tunable photo absorption window, longer excitons diffusion length, and anisotropic crystal morphology, are promising candidates to be added to improve the photon to current conversion efficiency (PCE) of OPV devices. Celik et al. have reported that the bulk-hetero junction (BHJ) hybrid solar cells based on CdSe nanorods (NRs) and the low band gap polymer poly[2,6-
(4,4-bis-(2-ethylhexyl)-4H-cyclopenta[2,1-b;3,4-b0]-dithiophene)-alt-4,7-(2,1,3-benzothiadiazole)] (PCPDTBT) have PCE about 3.5\% [8]. Moreover, the excitons transportation of direction in semiconductor NCs with anisotropic morphologies can improve PCE of polymer-based solar cell [8]. A high short-circuit current density $\left(J_{\mathrm{SC}}\right)$ can be achieved, which is attributed to the improved CdSe NRs surface modification resulting in lower recombination losses and better charge transport within the inorganic percolation network of the hybrid film [8].

The NCs with different morphologies and novel optical, electronic, and mechanical properties can be prepared by solution-grown methods [9-12]. Currently, NCs are used as electron acceptors in the hybrid $\mathrm{NC} /$ polymer solar cells [13]. When increasing the Se content in CdSeTe tetrapods (TPs), the band level positions between the polymer donor and the NCs acceptor may be modified, thereby promoting the open circuit voltage $\left(V_{\mathrm{OC}}\right)$ and $J_{\mathrm{SC}}$ [13]. Furthermore, the use of tetrapod-shaped NCs is an attractive route to obtain high efficiencies through the promotion of electron transport perpendicular to the plane of the film [14]. CdSe NCs acting as electron-transporting and hole-blocking layers can substantially improve cell performance [15]. When CdSe 
NCs with different morphologies are blended with poly(3hexylthiophene) (P3HT), rod-like structures can provide a pathway for the electrons to move away from the dissociation site, which cause enhanced carrier lifetimes and increased devices efficiencies [16]. On the other hand, for CdSe nanoparticles (NPs), due to the lack of dimensionality for charge separation at the polymer/CdSe interface, the performance of CdSe:P3HT devices is poor [16].

The morphology of CdSe NCs can be controlled by reaction temperatures, reaction time, and $\mathrm{Cd} / \mathrm{Se}$ atomic ratios [17]. Besides, when seeded growth method is used, the structure of the CdSe seeds determines the morphology of CdSe/CdS nanoheterostructures. The CdSe NCs with wurtzite structure seed the formation of CdSe/CdS NRs whereas CdSe with zinc-blended structure initiates the growth of CdSe/CdS TPs [18]. Wurtzite CdS NR and zinc-blended CdSe extensions can also be synthesized by isolation of the first-generation NCs and reintroduction of Cd precursor before injecting the second anion precursor [19].

In this study, we try to investigate the morphological effect of hybrid NRs and TPs on the conversion efficiency of PV cell. CdSe TPs are prepared by seed growth method and zinc-blended CdSe NCs are used as seeds to grow wurtzite CdSe arms. The CdSe NCs dissolved into dichlorobenzene (DCB) and mixed with P3HT: [6, 6]-phenyl-C61-butyric acid methyl ester (PCBM) are used as the active layer of the organic photovoltaic devices (OPV) devices.

The morphology, structure, optical properties, and efficiency detection are analyzed by transmission electron microscopy (TEM), X-ray diffraction (XRD), UV-visible absorption spectroscopy (UV-vis), fluorescence (FL), high fidelity solar simulator, and IV measurement (IV), respectively.

\section{Experimental Procedure}

2.1. Preparation of CdSe NCs. $2 \mathrm{mmol}$ of $\mathrm{CdO}$ and $4 \mathrm{mmol}$ of stearic acid (SA) were mixed in $5 \mathrm{~mL}$ 1-octadecene (ODE) and heated to $200^{\circ} \mathrm{C}$ under Ar. After the solution became clear, the temperature was reduced to RT. $37 \mathrm{~mL}$ ODE and $1 \mathrm{mmol}$ of Se powder were then added and the temperature was raised from RT to $240^{\circ} \mathrm{C}$ with the rise rate of $8^{\circ} \mathrm{C} / \mathrm{min}$ to grow the CdSe seeds. After the temperature reached $240^{\circ} \mathrm{C}$, the solution was harvested at room temperature and by washing with methanol it was stored in hexane and named as CdSe-NP. The concentration of the NPs in hexane is about $0.115 \mathrm{M}$. After that, $3 \mathrm{~mL}$ of NPs solution was mixed with $0.4 \mathrm{mmol}$ of Se powders and $1 \mathrm{~mL}$ of tri-n-octylphosphine (TOP) as the Se precursor, and Se precursor was injected into $\mathrm{Cd}$ precursor of a mixture of $0.8 \mathrm{mmol}$ of $\mathrm{CdO}, 1.6 \mathrm{mmol}$ of n-hexylphosphonic acid (HPA), and $1.7344 \mathrm{~g}$ of tri-noctylphosphine oxide (TOPO) at $300^{\circ} \mathrm{C}$, when the reaction time increases to 60 mins. After that the sample was cooled down to room temperature immediately and purified by hot anhydrous methanol at $70^{\circ} \mathrm{C}$ to remove residual surfactants and named as CdSe-TP.

2.2. Preparation of OPV Devices. Firstly, the ITO surface was exposed to UV-ozone for $10 \mathrm{~min}$. Then, a PEDOT: PSS layer was deposited by spin coating with a spin rate of $5000 \mathrm{rpm}$ for $50 \mathrm{sec}$ in order to increase its conductivity and then dried on hot plate at $120^{\circ} \mathrm{C}$ for $10 \mathrm{~min}$ in air. After cooling to room temperature, the ITO glasses were taken into the glove box in high purity nitrogen atmosphere. $\mathrm{P} 3 \mathrm{HT}$ and PCBM were dissolved into $0.7 \mathrm{~mL}$ dichlorobenzene (DCB) with the weight ratio of $1: 1$ as the standard active layer solution, and the prepared samples, CdSe TP/NR, were dissolved in the standard active layer solution with the concentration of 10 , 30, 50, 100, and $175 \mathrm{wt} \%$ and named as CdSe-TP/NR1, 2, 3, 4, and 5, respectively. Moreover, CdSe NP with $50 \mathrm{wt} \%$ was also prepared for comparison. The active layer solution was then coated on the PEDOT: PSS layer by spin coating $(500 \mathrm{rpm}$ for $50 \mathrm{sec}$ and then $1600 \mathrm{rpm}$ for $1 \mathrm{sec}$ ). After that, the ITO glasses were put into the petri dish to have solvent annealing until the surface color turned from yellow to dark purple. The ITO glasses were dried on the hot plate at $150^{\circ} \mathrm{C}$ for $30 \mathrm{~min}$ and cooled down to room temperature. Afterwards the ITO glasses were scratched as a part of the PEDOT: PSS and active layer as the anode. Then the ITO glasses were put on the mask and taken into high vacuum chamber and $\mathrm{Ca}$ and $\mathrm{Al}$ were evaporated and deposited on the ITO surface as the cathode. In standard device, the thickness of PEDOT:PSS, P3HT:PCBM, and Ca/Al layer is 40,300 , and $50 / 90 \mathrm{~nm}$, respectively. The active area of all devices is $0.5 \times 0.2 \mathrm{~cm}^{2}$. The device architecture and the energy band diagram of the CdSe NCs modified OPV cells are illustrated in Figure 1. Based on the energy band diagram we know that the CdSe NCs are promising candidates to transport the electron from PCBM to $\mathrm{Al} / \mathrm{Ca}$ cathode.

2.3. Characterization. Optical absorption spectra were carried out by UV-visible absorption spectrophotometer (UVvis) at room temperature (Thermo Fisher Scientific, Evolution $60 \mathrm{~s}$ ). The light emission spectra of CdSe NCs were determined at room temperature by fluorescence (FL, Hitachi F-7000). Emission spectra were taken by using an excitation wavelength of $365 \mathrm{~nm}$ and a slit width of $2.5 \mathrm{~nm}$. The measurements were carried out in the range of $450-700 \mathrm{~nm}$.

The morphologies of CdSe NCs were determined by high resolution transmission electron microscopy (HRTEM, JEOL, JEM-2100). The purified NCs were dispersed in hexane and the solution was dropped on a 300-mesh carbon-coated copper grid and then put into oven $\left(100^{\circ} \mathrm{C}\right)$ for $2 \mathrm{hrs}$ followed by natural evaporation at room temperature. The phases and structures of CdSe NCs were detected by X-ray diffraction (XRD) with $\mathrm{Cu} \mathrm{K} \alpha$ radiation. A Guinier powder diffractometer (Huber, Germany), set at $45^{\circ}$ transmission angle, was used in this work. The purpose of solar simulator was to provide a controllable indoor test facility under laboratory conditions.

The standard of solar simulator (Newport, 69911, 95$264 \mathrm{VAC}, 50 / 60 \mathrm{~Hz}, 8 \mathrm{AMPS}$ ) for the detection of solar cell was under AM $1.5 \mathrm{G}$ and $100 \mathrm{~mW} / \mathrm{cm}^{2}$. The detection of IV curves for solar cell was measured by the IV measurement (IV, Keithley semiconductor characterization system 4200SCS and Keithley interactive test environment software). The light source in this case was a class A quality solar simulator (PECeL11, AM1.5G, Peccell Technologies Inc.); light 


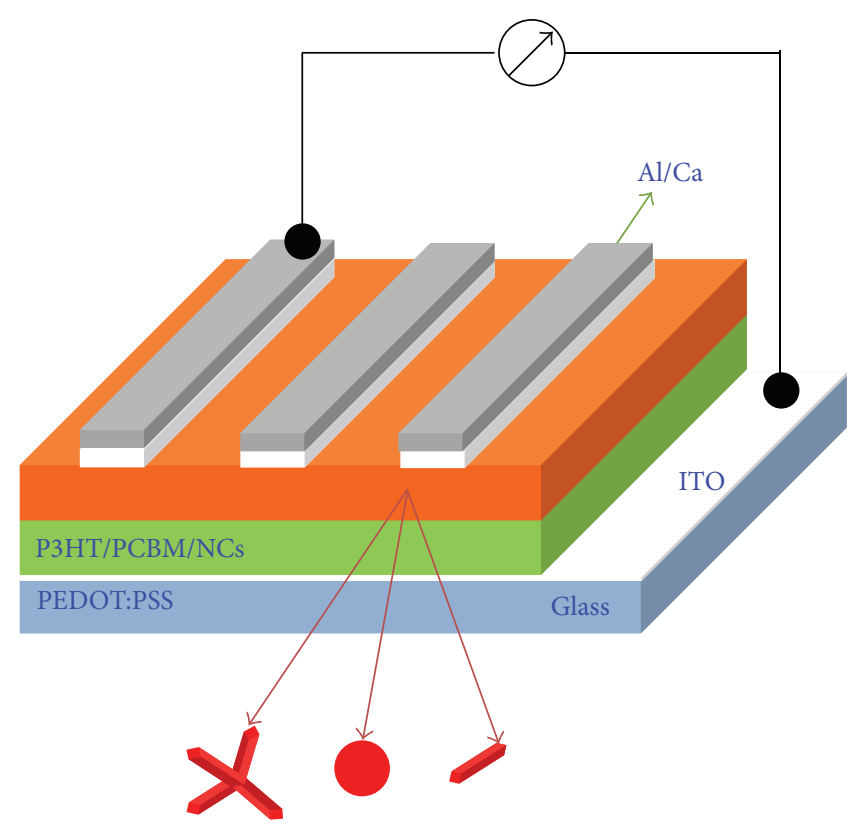

(a)
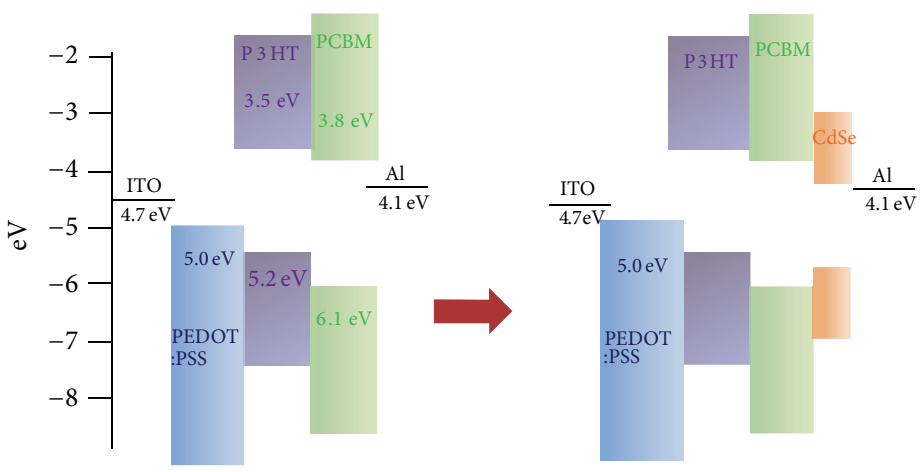

(b)

FIGURE 1: The device architecture and the energy band diagram of the CdSe NCs-modified OPV cells.

was focused through a monochromator (Oriel Instrument, Stratford, CT, model 74100) onto the photovoltaic cell. The monochromator was moved in steps from $300 \mathrm{~nm}$ to $900 \mathrm{~nm}$ (through the visible spectrum) to generate the incident photon to current conversion efficiency (IPCE) as defined in

$$
\operatorname{IPCE}(\%)=1240\left(\frac{J_{\mathrm{SC}}}{\lambda \varphi}\right),
$$

where $\lambda$ is the wavelength, $J_{\mathrm{SC}}$ is the short-circuit photocurrent density $\left(\mathrm{mA} / \mathrm{cm}^{2}\right)$ recorded using a potentiostat/galvanostat, and $\varphi$ is the incident radiative flux $\left(\mathrm{W} / \mathrm{m}^{2}\right)$ measured using an optical detector (Oriel Instrument, model 71580) and power meter (Oriel Instrument, model 70310) [20].

\section{Results and Discussion}

3.1. The Physical Properties of CdSe NCs. Since the absorption and emission properties of semiconductor NCs affect the efficiency of OPV, two basic methods, including extending the absorption window and reducing emission intensity of NCs, have been applied to improve the PCE of devices [21]. The UV-vis and FL spectra of spherical and anisotropic CdSe NCs are compared in Figure 2. Due to the stronger quantum confinement effect for CdSe-NP, their absorption and emission peaks are obvious, implying that electronhole (e-h) separation and recombination efficiency are good. However, this significant emission behavior for the CdSeNP sample means that the carrier transport to positive and negative electrodes is poor, which suggests that this CdSeNP may not have positive effect on the device efficiency when used as acceptor materials. As we have mentioned above that, based on the energy band diagram shown in Figure 1, the CdSe NC can be used as acceptor materials to promote the transportation of the electrons from PCBM to Al/Ca cathode. If the carrier is recombined in CdSe NP, a decrease in the concentration of $(\mathrm{e}-\mathrm{h})$ pairs and the number of carriers will be observed in the CdSe NP-modified OPV devices.

On the other hand, in Figure 2, although the absorption edge of CdSe TP/NR is not obvious, its absorption window is 


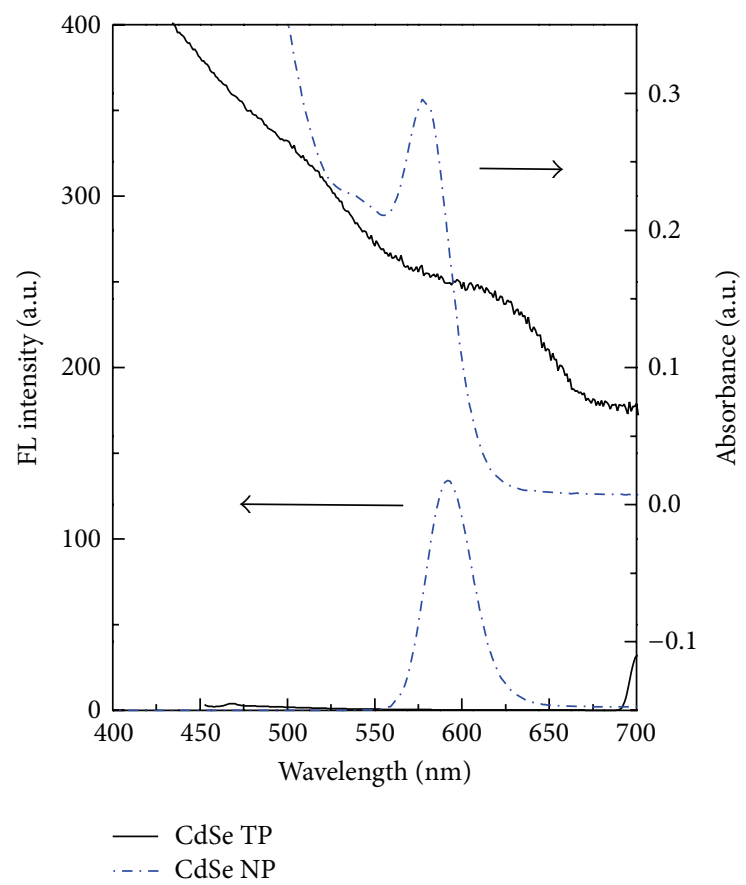

Figure 2: The UV-vis and FL spectra of CdSe NCs.

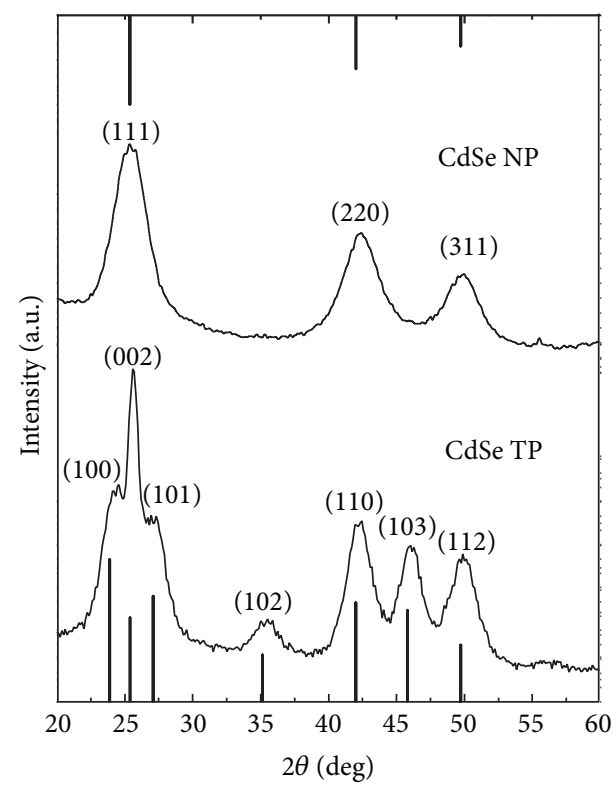

FIGURE 3: The XRD patterns of CdSe NCs.

longer than that of CdSe NP. Moreover, the emission intensity of CdSe-NP is much more obvious than that of CdSeTP/NR. Since the emission phenomenon of CdSe-TP/NT is suppressed, the sample may not form effective e-h pairs, meaning that the carrier separation may not be effective and e-h pair recombination is suppressed. Therefore, CdSe-NP can effectively form excitons, suggesting that it has better carrier separation efficiency. Because of the large absorption window and lower recombination efficiency in CdSe TP/NR sample, it is expected that the carrier transport to cathode and anode in this sample is more effectively than that in CdSe NP sample, and the device efficiency of OPV may be improved by combining CdSe TP/NR as donor material. It has been reported that the NCs with different morphologies have a tendency to form directed chains with the particles stacking along their long axis, which can promote the PCE [21].

Figure 3 displays the XRD patterns of CdSe NCs. The peaks of CdSe NP are located at 25.36, 42.01, and $49.72^{\circ}$ which belong to (111), (220), and (311) planes of zincblended structure (JCPDS 88-2346), respectively. Based on thermodynamics, it has been reported that zinc blend is the most stable form at lower temperature, while wurtzite is more stable in high temperature [20]. For CdSe TP/NR, peaks located at 23.88, 25.39, 27.09, 35.13, 41.99, 45.81, and $49.71^{\circ}$ corresponding to wurtzite structure of CdSe (JCPDS 770046) are observed, proving that wurtzite arms successfully cover the zinc-blended seeds. It is noted that (002) plan is the preferred orientation and this anisotropic structure of NCs might be beneficial for the conversion efficiency of OPV devices.

Figure 4 displays the morphologies of CdSe NCs by HRTEM. The CdSe NP has spherical shape with an average particle size of $3.6 \pm 0.3 \mathrm{~nm}$. On the other hand, the tetrapod and rod-like morphologies are observed with an average length of the arms about $14.0 \mathrm{~nm}$ when using HPA and TOPO as cosolvents. The ratio between TP and NR is about 2.3 in the CdSe TP/NR sample. It is reported that HPA molecule can absorb certain crystal surface of CdSe, thereby promoting the same direction growth and resulting in anisotropic morphology [22]. Moreover, based on the above characterizations, it can be summarized that CdSe TP/NR with longer absorption window, lower carrier recombination efficiency, and anisotropic shape may improve the OPV efficiency.

3.2. The Efficiency of OPV Devices Modified by CdSe NC with Various Concentrations. In order to understand the absorption phenomenon of the active layer, the absorbance of CdSe NP and CdSe TP/NR and standard samples under different wavelengths are compared in Figure 5. The absorption curves of CdSe TP/NR and standard sample are very similar in which the absorbance increases slightly in the range from 570 to $610 \mathrm{~nm}$ when the P3HT/PCBM is combined with CdSe NCs. Furthermore, although the band gap of CdSe NCs is smaller than that of P3HT/PCBM, the absorption window is not changed significantly. As a result, when the CdSe NCs are mixed with $\mathrm{P} 3 \mathrm{HT} / \mathrm{PCBM}$ as active layer, the UV-vis spectra are almost the same for all samples, meaning that the light absorption is similar for standard and CdSe NCs modified samples.

As we know that carrier transport efficiency affects the device performance [21], based on previous discussion, although the CdSe TP/NR possesses larger absorption window, poor (e-h) recombination efficiency, preferable orientation, and anisotropic morphology, its UV-vis spectra are almost the same with standard active layer material, meaning that adding CdSe NC may not have positive effect on the 

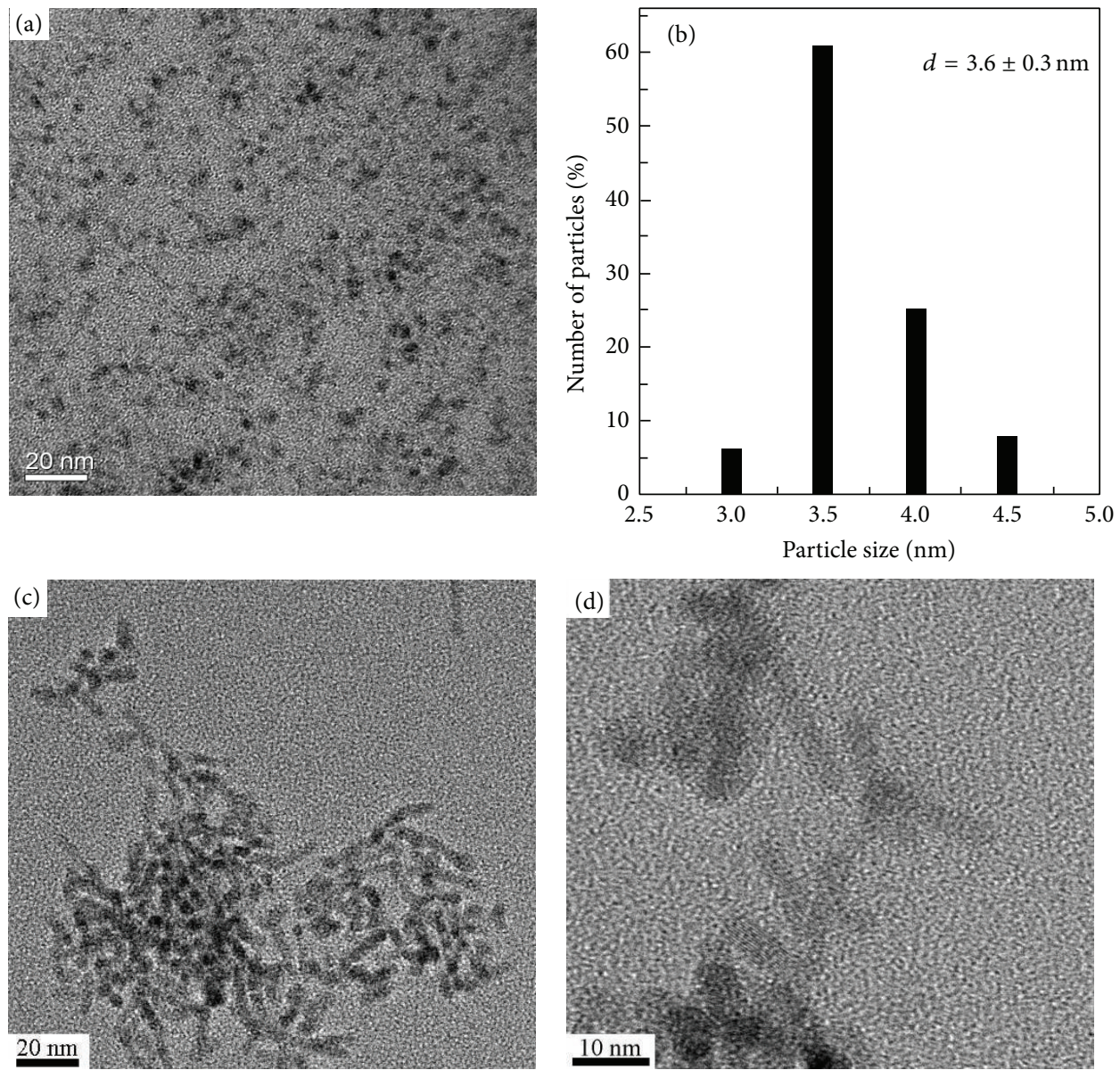

FIgURE 4: (a) TEM micrographs and (b) size distribution of CdSe NP. (c) and (d) are TEM micrographs of CdSe TP/NR.

light absorption and the possibility of IPCE enhancement stemming from light absorption can be ruled out. On the other hand, since the electron mobility in inorganic crystals is faster than that in organic material, the electron transport phenomena may be modified by the addition of CdSe NC, which can also have a chance to promote IPCE. In order to understand the carrier transfer between P3HT/PCBM and CdSe NC modified active layers, the IPCE and device performance of CdSe NP and TP/NR and standard samples are shown in Figure 6 and listed in Table 1. IPCE of the device can be improved by modification of CdSe TP/NR sample in the range of 400 and $600 \mathrm{~nm}$ and is decreased significantly by modification with CdSe-NP sample, confirming that anisotropic morphology can benefit the transport of the carrier, but the carrier is consumed and recombined in CdSe NP. Compared to the CdSe NP-modified device, the PCE enhancement is mainly due to the increased $J_{\mathrm{SC}}$. Suggesting that through the addition of a suitable amount of elongated TPs/NRs inorganic NC phase, a better connection between PCBM and NCs can be achieved, benefitting the electron transport to the negative electrode.

In order to optimize the concentration of CdSe TP/NR for the modification of the OPV device, the efficiencies of standard sample and CdSe TP/NR modified devices with various concentrations from 10 to $175 \mathrm{wt} \%$ are compared in Table 2 and Figure 7. Among them, the CdSe-TP/NR3 sample has the largest efficiency, suggesting that using CdSe TP/NR with an appropriate concentration to modify the active layer can significantly promote the efficiency by about $4.3 \%$ and suggesting an enhancement of $13.2 \%$ as listed in Table 2. When the concentration increases from 0 to 10,30 , and $50 \mathrm{wt} \%, J_{\mathrm{SC}}$ increases from 9.6 to $9.8,10.1$, and $10.9 \mathrm{~mA} / \mathrm{cm}^{2}$, and the efficiency increases from 3.80 to $3.86,4.01$, and $4.30 \%$, respectively. However, when the concentration further increases to 100 and $175 \mathrm{wt} \%, J_{\mathrm{SC}}$ decreases to 9.6 and $9.4 \mathrm{~mA} / \mathrm{cm}^{2}$ and the efficiency decreases to 3.58 and $3.19 \%$, respectively. Similar trend has been observed in the CdSe TPs and poly(2-methoxy-5-(2-ethylhexyloxy)-1,4-phenylene vinylene)(MEH-PPV) system [13]. When the weight ratio of CdSe:MEH-PPV increases from 1:2 to $9: 1$, the efficiency increases from 0.13 to $1.13 \%$, but when the weight ratio further increases from $9: 1$ to $20: 1$, the efficiency then decreases from 1.13 to $0.16 \%$. It seems that increasing CdSe content in the active layer will generate more percolation pathways and help the transport of electrons, thereby increasing the photocurrent and efficiency [13]. However, further increasing $\mathrm{CdSe}$ in the active layer decreases the contribution of polymer to the light absorption and destroys the pathway for holes 
TABLE 1: The $J_{S C}, V_{\mathrm{OC}}$, FF, efficiency, and enhancement of the standard sample, CdSe-NP, and CdSe-TP/NR3 added OPV device.

\begin{tabular}{lccccc}
\hline Device & $J_{\mathrm{SC}}\left(\mathrm{mA} / \mathrm{cm}^{2}\right)$ & $V_{\mathrm{OC}}(\mathrm{V})$ & FF & Efficiency $(\%)$ & Improvement $(\%)$ \\
\hline Standard & 9.6 & 0.63 & 0.63 & 3.80 & 0 \\
CdSe-NP & 9.0 & 0.60 & 0.60 & 3.26 & -14.2 \\
CdSe-TP/NR3 & 10.9 & 0.64 & 0.62 & 4.30 & 13.2 \\
\hline
\end{tabular}

TABLE 2: The $J_{\mathrm{SC}}, V_{\mathrm{OC}}, \mathrm{FF}$, efficiencies, and enhancement of the standard sample and CdSe-TP/NR modified OPV device with different concentrations.

\begin{tabular}{lcccccc}
\hline Device & Concentrations $(\mathrm{wt} \%)$ & $J_{\mathrm{SC}}\left(\mathrm{mA} / \mathrm{cm}^{2}\right)$ & $V_{\mathrm{OC}}(\mathrm{V})$ & FF & Efficiency $(\%)$ & Improvement $(\%)$ \\
\hline Standard & 0 & 9.6 & 0.63 & 0.63 & 3.80 & 0 \\
CdSe-TP/NR1 & 10 & 9.8 & 0.62 & 0.63 & 3.86 & 1.5 \\
CdSe-TP/NR2 & 30 & 10.1 & 0.63 & 0.63 & 4.01 & 5.5 \\
CdSe-TP/NR3 & 50 & 10.9 & 0.64 & 0.62 & 4.30 & 3.58 \\
CdSe-TP/NR4 & 100 & 9.6 & 0.62 & 0.60 & 3.2 & -5.8 \\
CdSe-TP/NR5 & 175 & 9.4 & 0.62 & 0.55 & 3.19 & -16.0 \\
\hline
\end{tabular}

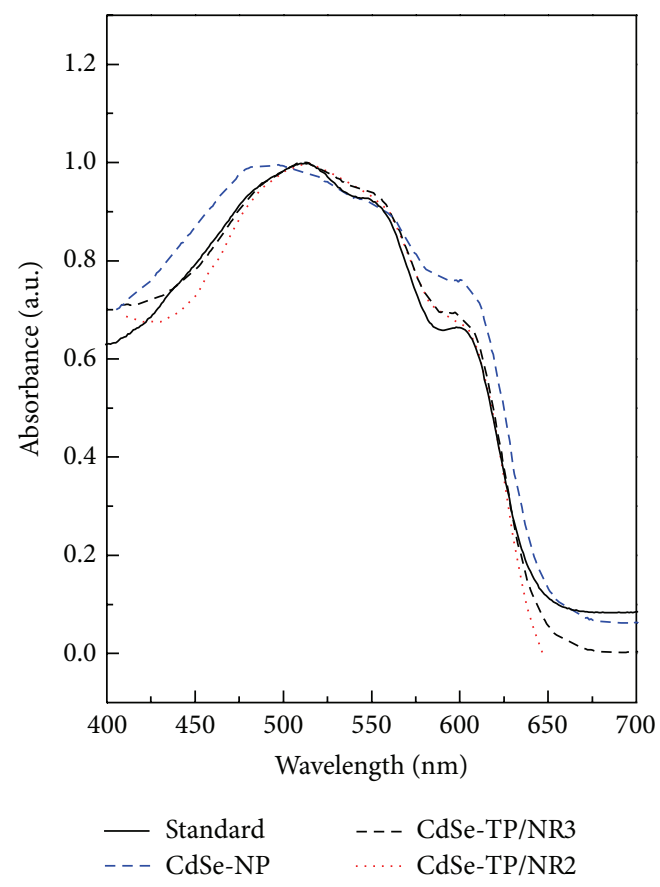

FIgURE 5: The UV-vis of the active layer on ITO glass.

transport. As a result, the efficiency decreases accordingly [13]. Zhou et al. have pointed out that PCE of blend CdSe NPs and NRs is higher than that of pure NPs and NRs [23]. Besides, higher NP concentration can enhance the PCE. It could be attributed to the improved conductivity of the active layer network due to the addition of suitable amounts of NPs and NRs, which provide more direct and continuous pathways for the electron transport. In our study the ratio between TP and NR is about 2.3 and we know that the TPs can offer more direct and continuous pathways for the electron transport, resulting in more effective power conversion of CdSe-TP/NR samples. Moreover, the optimal concentration of CdSe in P3HT: PCBM system should be $50 \mathrm{wt} \%$.

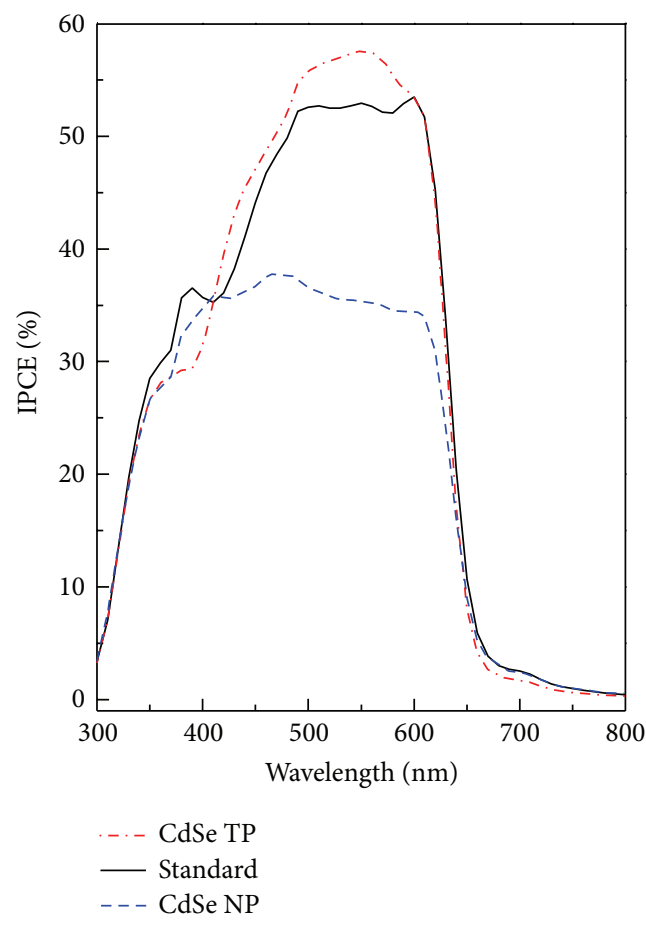

FIGURE 6: IPCE spectra of OPV devices used different addition in active layer.

It is worth mentioning that, in Figure 7, CdSeTP/NR3device performs a $J_{\mathrm{SC}}$ of $10.9 \mathrm{~mA} / \mathrm{cm}^{2}$, implying that the EQE should be $>75 \%$. However, the real EQE displayed in Figure 6 is obviously lower than $75 \%$. Generally, the EQE and convention efficiency of devices cannot be measured simultaneously. If $\mathrm{Ca} / \mathrm{Al}$ electrode is evaporated after $\mathrm{EQE}$ measurement, the efficiency decreases very significantly. Since the device is prepared under gloves box, the environmental effect can be ignored and similar trends of EQE and $J_{\mathrm{SC}}$ for various samples are obtained in this study.

Moreover, it has been reported that when the aspect ratio of the NRs increases from 1 to 10 , the charge transport can 


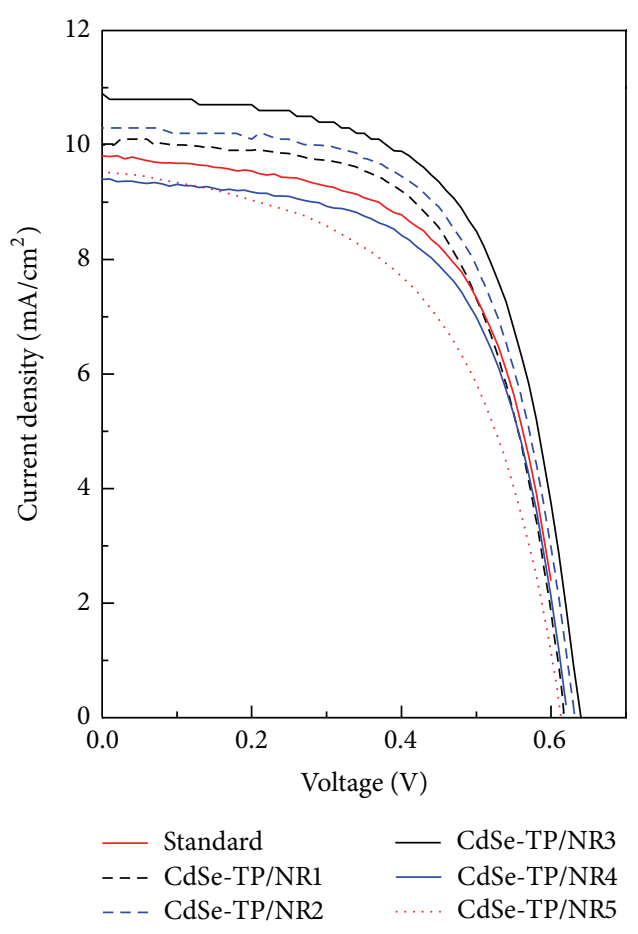

Figure 7: The $J-V$ curves of CdSe TP/NR modified devices.

be improved substantially and an EQE enhancement is noted [24]. The cell performance is strongly dependent on blend morphology, and solution-based fabrication techniques often cause uncontrolled and irreproducible blends. As a result, the cell performance of blending with hyperbranched CdSe NCs is better than that of NRs [12]. In principle, elongated or branched NCs such as NRs and TPs can provide more extended electrical conductive pathways, because the number of interparticle hopping events for extracting electrons towards the electrode is reduced. Therefore, it is reported that, for pyridine capped CdSe NCs, devices with NRs and TPs usually exhibit better performance than QDs based devices [24]. However, NRs tend to be horizontally aligned instead of being aligned vertically to the film. In such case, elongated structures are not superior to spherical QDs for extracting free carriers since the electrons need to hop in the vertical direction from the exciton dissociation site to the cathode. It seems that if we can make the larger size of CdSe-NR/TP NCs perpendicular on the PEDOT:PSS film preciously, it will be more beneficial to the charge transport and the enhancement of PCE of devices.

\section{Conclusions}

In this study, CdSe NCs with spherical-like and tetrapod/rodlike morphologies have been prepared and applied as the donor in the active layer of the OPV devices to increase their photoelectron conversion efficiency. Seed growth synthesis is used for the preparation of CdSe TP/NR samples with a wurtzite arms length of $14.0 \mathrm{~nm}$ covering the zinc-blended seeds.
For the devices with concentrations of CdSe TP/NR from 0 to $50 \mathrm{wt} \%, J_{\mathrm{SC}}$ is increased from 9.6 to $10.9 \mathrm{~mA} / \mathrm{cm}^{2}$, and efficiency is promoted from 3.80 to $4.30 \%$, respectively, due to the generation of pathways for electrons transport. However, further increasing of the concentration to 100 and $175 \mathrm{wt} \%$ decreases the contribution of polymer to the light absorption and destroys the pathway for holes transport. As a result, $J_{\mathrm{SC}}$ and efficiency are declined accordingly.

\section{Conflict of Interests}

The authors declare that there is no conflict of interests regarding the publication of this paper.

\section{Authors' Contribution}

Shu-Ru Chung and Kuan-Wen Wang have contributed equally to this work.

\section{Acknowledgment}

This work was supported by the National Science Council of Taiwan under Contract nos. 102-2221-E-008-030, 102-2120M-007-007, and 102-2218-E-150-001.

\section{References}

[1] Y. Chang and L. Wang, "Efficient poly(3-hexylthiophene)-based bulk heterojunction solar cells fabricated by an annealing-free approach," Journal of Physical Chemistry C, vol. 112, no. 45, pp. 17716-17720, 2008.

[2] X. Wang, T. Ishwara, W. Gong, M. Campoy-Quiles, J. Nelson, and D. D. C. Bradley, "High-performance metal-free solar cells using stamp transfer printed vapor phase polymerized poly $(3,4-$ ethylenedioxythiophene) top anodes," Advanced Functional Materials, vol. 22, no. 7, pp. 1454-1460, 2012.

[3] W. Li, A. Furlan, K. H. Hendriks, M. M. Wienk, and R. A. J. Janssen, "Efficient tandem and triple-junction polymer solar cells," Journal of the American Chemical Society, vol. 135, pp. 5529-5532, 2013.

[4] J. M. Lobez, T. L. Andrew, V. Bulović, and T. M. Swager, "Improving the performance of P3HT-fullerene solar cells with side-chain-functionalized poly(thiophene) additives: a new paradigm for polymer design," ACS Nano, vol. 6, no. 4, pp. 3044-3056, 2012.

[5] K. Foe, G. Namkoong, M. Samson, E. M. Younes, I. Nam, and T. M. Abdel-Fattah, "Stability of high band gap P3HT : PCBM organic solar cells using $\mathrm{TiO}_{x}$ interfacial layer," International Journal of Photoenergy, vol. 2014, Article ID 784724, 6 pages, 2014.

[6] Z. He, C. Zhong, S. Su, M. Xu, H. Wu, and Y. Cao, "Enhanced power-conversion efficiency in polymer solar cells using an inverted device structure," Nature Photonics, vol. 6, pp. 591-595, 2012.

[7] S. K. Park, Y. H. Kim, and J. I. Han, "Effect of annealing treatment and surface morphology on power conversion in organic photovoltaics," Japanese Journal of Applied Physics, vol. 48, no. 8R, Article ID 081505, 3 pages, 2009.

[8] D. Celik, M. Krueger, C. Veit et al., "Performance enhancement of CdSe nanorod-polymer based hybrid solar cells utilizing 
a novel combination of post-synthetic nanoparticle surface treatments," Solar Energy Materials and Solar Cells, vol. 98, pp. 433-440, 2012.

[9] Y.-W. Jun, S.-M. Lee, N.-J. Kang, and J. Cheon, "Controlled synthesis of multi-armed CdS nanorod architectures using monosurfactant system," Journal of the American Chemical Society, vol. 123, no. 21, pp. 5150-5151, 2001.

[10] L. Fang, J. Y. Park, Y. Cui et al., "Mechanical and electrical properties of CdTe tetrapods studied by atomic force microscopy," Journal of Chemical Physics, vol. 127, no. 18, Article ID 184704, 2 pages, 2007.

[11] T. Mokari, E. Rothenberg, I. Popov, R. Costi, and U. Banin, "Selective growth of metal tips onto semiconductor quantum rods and tetrapods," Science, vol. 304, no. 5678, pp. 1787-1790, 2004.

[12] I. Gur, N. A. Fromer, C. Chen, A. G. Kanaras, and A. P. Alivisatos, "Hybrid solar cells with prescribed nanoscale morphologies based on hyperbranched semiconductor nanocrystals," Nano Letters, vol. 7, no. 2, pp. 409-414, 2007.

[13] Y. Zhou, Y. Li, H. Zhong et al., "Hybrid nanocrystal/polymer solar cells based on tetrapod-shaped CdSe $\mathrm{Te}_{1-x}$ nanocrystals," Nanotechnology, vol. 17, no. 16, pp. 4041-4047, 2006.

[14] B. Sun, E. Marx, and N. C. Greenham, "Photovoltaic devices using blends of branched CdSe nanoparticles and conjugated polymers," Nano Letters, vol. 3, no. 7, pp. 961-963, 2003.

[15] J. H. Jeon, D. H. Wang, H. Park, J. H. Park, and O. O. Park, "Stamping transfer of a quantum dot interlayer for organic photovoltaic cells," Langmuir, vol. 28, no. 25, pp. 9893-9898, 2012.

[16] S. Dayal, M. O. Reese, A. J. Ferguson, D. S. Ginley, C. Rumbles, and N. Kopidakis, "The effect of nanoparticle shape on the photocarrier dynamics and photovoltaic device performance of poly(3-hexylthiophene):CdSe nanoparticle bulk heterojunction solar cells," Advanced Functional Materials, vol. 20, no. 16, pp. 2629-2635, 2010.

[17] Z. A. Peng and X. Peng, "Nearly monodisperse and shapecontrolled CdSe nanocrystals via alternative routes: nucleation and growth," Journal of the American Chemical Society, vol. 124, no. 13, pp. 3343-3353, 2002.

[18] D. V. Talapin, J. H. Nelson, E. V. Shevchenko, S. Aloni, B. Sadtler, and A. P. Alivisatos, "Seeded growth of highly luminescent $\mathrm{CdSe} / \mathrm{CdS}$ nanoheterostructures with rod and tetrapod morphologies," Nano Letters, vol. 7, no. 10, pp. 2951-2959, 2007.

[19] D. Milliron, S. M. Hughes, Y. Cui et al., "Colloidal nanocrystal heterostructures with linear and branched topology," Nature, vol. 430, no. 6996, pp. 190-195, 2004.

[20] Z. Deng, L. Cao, F. Tang, and B. Zou, "A new route to zincblende CdSe nanocrystals: mechanism and synthesis," Journal of Physical Chemistry B, vol. 109, no. 35, pp. 16671-16675, 2005.

[21] W. U. Huynh, X. Peng, and A. P. Alivisatos, "CdSe nanocrystal rods/poly(3-hexylthiophene) composite photovoltaic devices," Advanced Materials, vol. 11, no. 11, pp. 923-927, 1999.

[22] K. P. Rice, A. E. Saunders, and M. P. Stoykovich, "Seedmediated growth of shape-controlled wurtzite CdSe nanocrystals: platelets, cubes, and rods," Journal of the American Chemical Society, vol. 135, no. 17, pp. 6669-6676, 2013.

[23] Y. Zhou, M. Eck, C. Men et al., "Efficient polymer nanocrystal hybrid solar cells by improved nanocrystal composition," Solar Energy Materials and Solar Cells, vol. 95, no. 12, pp. 3227-3232, 2011.
[24] W. U. Huynh, J. J. Dittmer, and A. P. Alivisatos, "Hybrid nanorod-polymer solar cells," Science, vol. 295, no. 5564, pp. 2425-2427, 2002. 

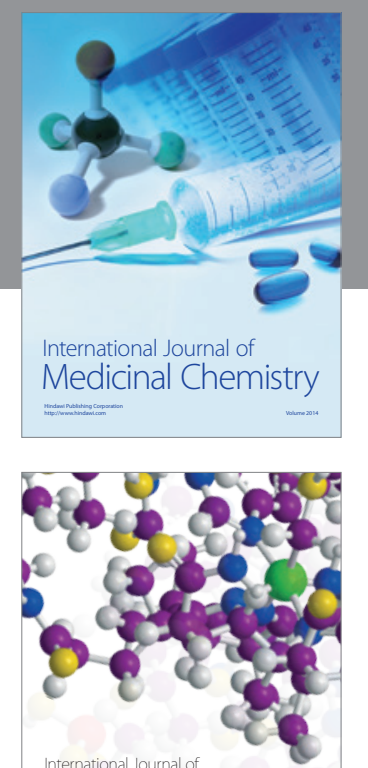

\section{Carbohydrate} Chemistry

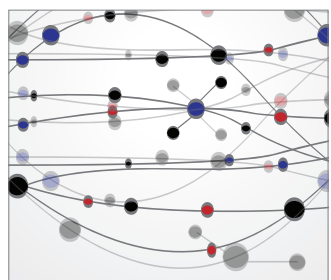

The Scientific World Journal
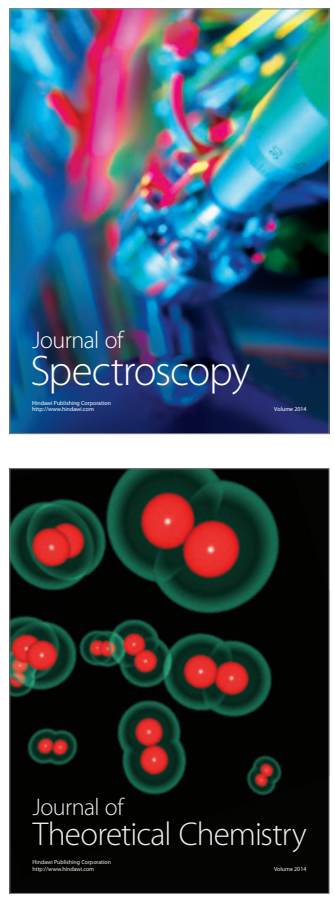
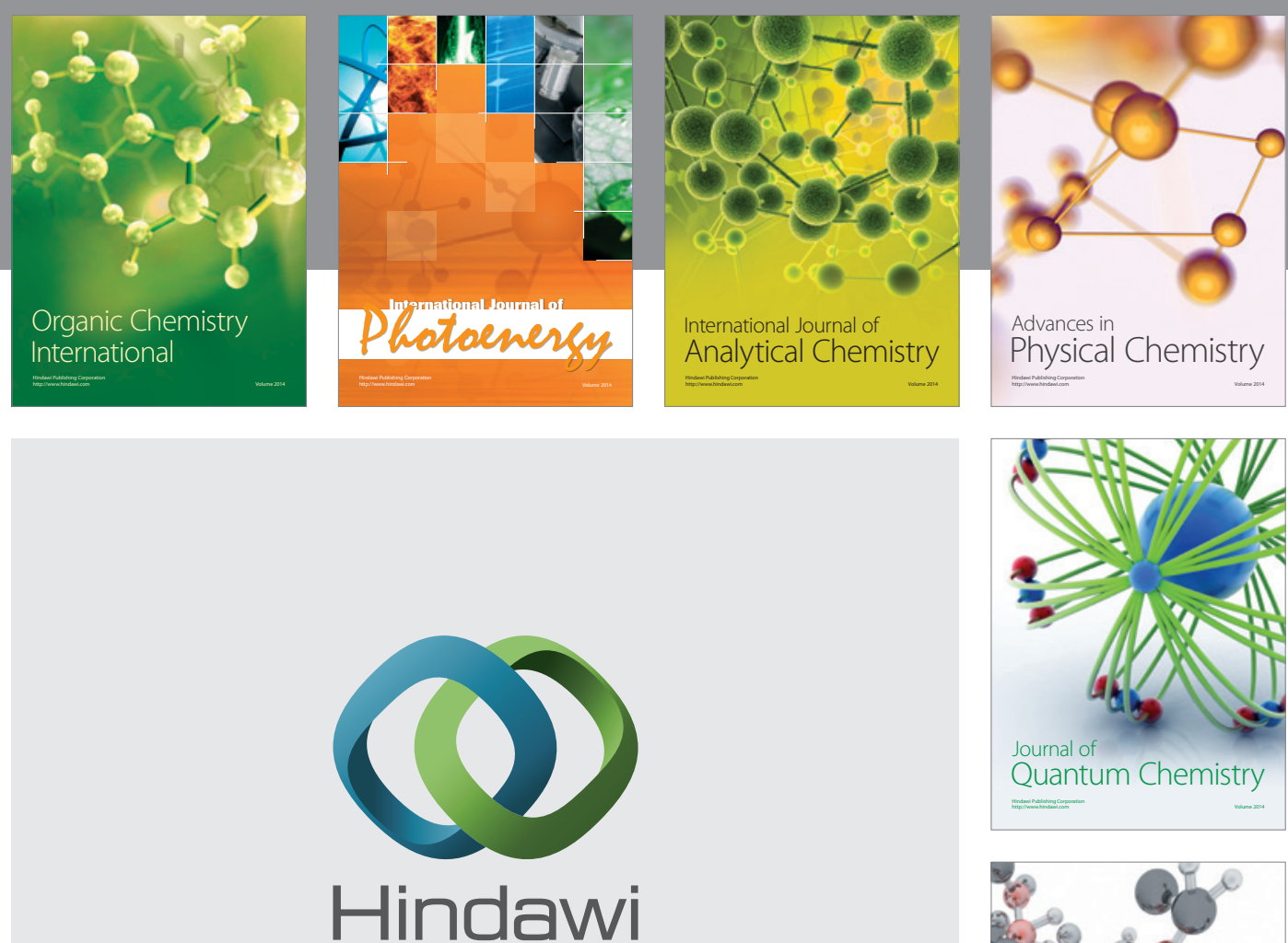

Submit your manuscripts at

http://www.hindawi.com

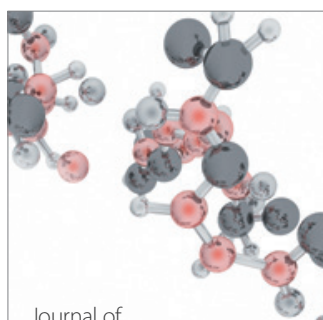

Analytical Methods

in Chemistry

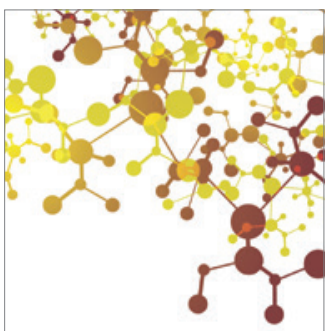

Journal of

Applied Chemistry

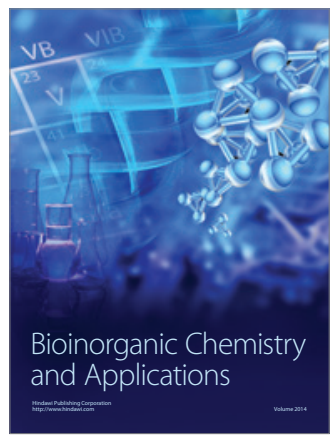

Inorganic Chemistry
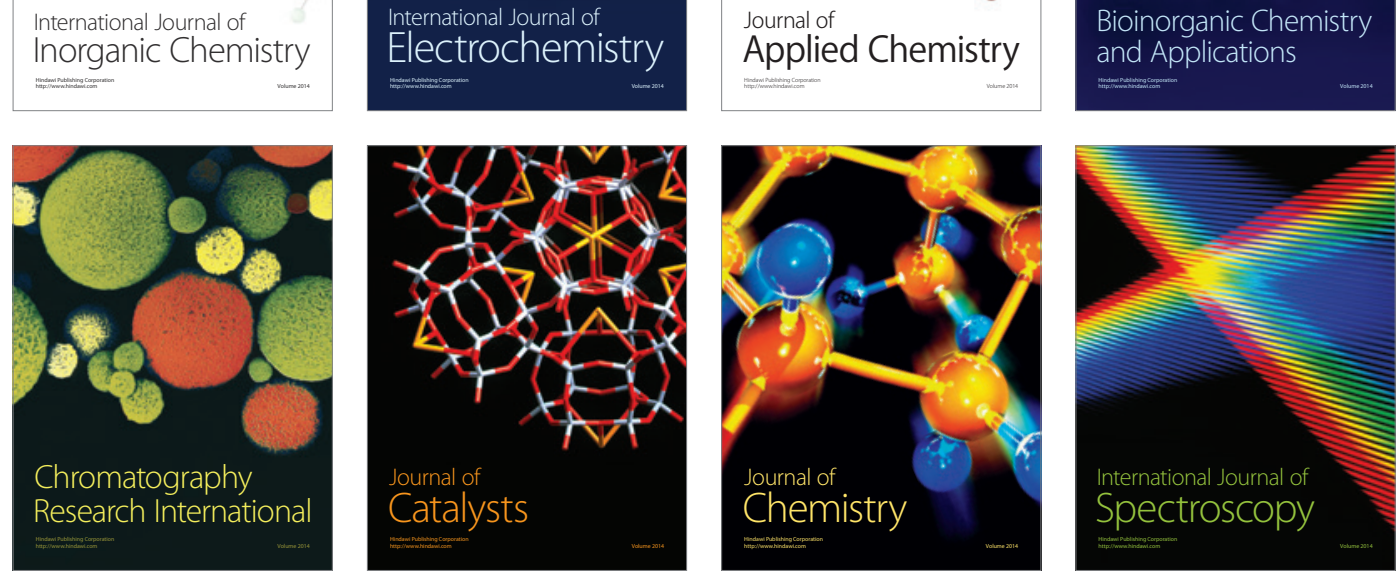